Sugang Ma, Fengquan Zhong* and Xinyu Zhang

\title{
Numerical Study of Unsteady Properties of Ethylene/Air Turbulent Jet Diffusion Flame with Detached Eddy Simulation
}

DOI 10.1515/tjj-2015-0045

Received July 29, 2015; accepted August 11, 2015

\begin{abstract}
In this paper, unsteady process of ignition and combustion of turbulent plane-jet diffusion flame of ethylene/air is numerically simulated with detached eddy simulation (DES) and a reduced kinetic mechanism of ethylene. The kinetic mechanism consisting of 25 species and 131 steps is reduced from a 25 species/131 steps detailed mechanism via the method of error-propagationbased directed relation graph (DRGEP). The DES results of averaged temperature profiles at varied downstream locations are compared with the DNS results of Yoo et al. [11] and satisfactory agreement between them is found. Ignition and combustion of ethylene plane-jet diffusion flame is simulated and dynamic changes of temperature field and $\mathrm{OH}$ radical are obtained. The present numerical study shows that DES method with a qualified reduced mechanism of hydrocarbon fuels can effectively simulate temporal and spatial evolution of ignition and combustion process.
\end{abstract}

Keywords: combustion, ignition, hydrocarbon fuel, reduced mechanism, detached eddy simulation

PACS $^{\circledR}$ (2010). 47.27.E-, 47.27.ep, 47.70.Pq

\section{Introduction}

Hydrocarbons such as methane, propane and ethylene are often used as fuel for air-breathing or rocket engines $[1,2]$. Compared to hydrogen, ethylene has a longer ignition delay time and lower peak flame speed, which greatly affect the ignition reliability and combustion

*Corresponding author: Fengquan Zhong, State Key Laboratory of High Temperature Gas Dynamics, Institute of Mechanics, Chinese Academy of Sciences, Beijing 100190, China, E-mail: fzhong@imech.ac.cn

Sugang Ma: E-mail: masugang12@mails.ucas.ac.cn, Xinyu Zhang: E-mail: changxy@imech.ac.cn, State Key Laboratory of High Temperature Gas Dynamics, Institute of Mechanics, Chinese Academy of Sciences, Beijing 100190, China performance. Therefore, it is imperative to study the unsteady process of ignition and combustion of ethylene flame.

Numerical simulation of combustion process of hydrogen or methane has been widely conducted $[3,4]$. For hydrocarbons with larger molecules than methane, the most critical issue required to be solved prior to simulation is how to handle the complicated kinetic mechanism of hydrocarbons. It is known that a good kinetic mechanism must contain hundreds or even thousands of species and elementary reactions for an accurate simulation of reaction processes, so direct applications of detailed mechanisms in numerical simulation, especially for turbulent combustion becomes infeasible. Hence, reduction of a detailed reaction mechanism into a smaller one that is acceptable for numerical simulation is a key issue for modeling of reacting flows.

In recent years, $\mathrm{Lu}$ and Law [5] proposed the directed relation graph (DRG) method for efficient mechanism reduction. The DRG method computes the contributions of other species to the consumption or production rate of the target species and deletes species with lesser contributions. Pepiot-Desjardins and Pitsch [6] and Niemeyer et al. [7] then improved the original DRG method and developed the directed relation graph with error propagation (DRGEP) method. It was shown that compared to DRG, DRGEP produces more compact mechanisms for the same level of accuracy. Zhong et al. [8] reduced the detailed mechanisms of ethylene, n-dodecane and surrogate of kerosene using DRGEP and sensitivity analysis and examined accuracy of the reduced mechanisms with varied species numbers.

In this paper, unsteady process of ignition and combustion of turbulent plane-jet diffusion flame of ethylene/ air is numerically simulated with detached eddy simulation (DES) and a reduced kinetic mechanism of ethylene. The reduced mechanism of ethylene (25 species and 131 steps) is obtained from a detailed mechanism of ethylene (71 species and 395 steps) proposed by Wang [9] with the reduction method of error-propagation-based directed relation graph (DRGEP) [7]. The present numerical study shows that DES method with the reduced mechanism of ethylene can effectively simulate temporal and spatial 
changes of flow field and species in the ignition and combustion process.

\section{Numerical methods}

Figure 1 shows a schematic diagram of the planar jet reactor with a 2-millimeter-wide slot in the center for fuel injection. The injection speed of ethylene fuel is $204 \mathrm{~m} / \mathrm{s}$ and the injection temperature is $550 \mathrm{~K}$. The air flow has an inlet speed of $20 \mathrm{~m} / \mathrm{s}$ and an inlet temperature of 1,550 K. The Navier-Stokes equations and transport equations of species are solved with finite volume method with a DES model based on Menter's SST k- $\omega$ model [10]. The convective terms of the equations are spatially discretized using a 2nd-order upwind scheme and the viscous terms are solved with a 2nd-order central scheme. The solution is advanced in time through an implicit Euler method. A perfect gas assumption is used and a turbulent Schmidt number is set to 0.7.

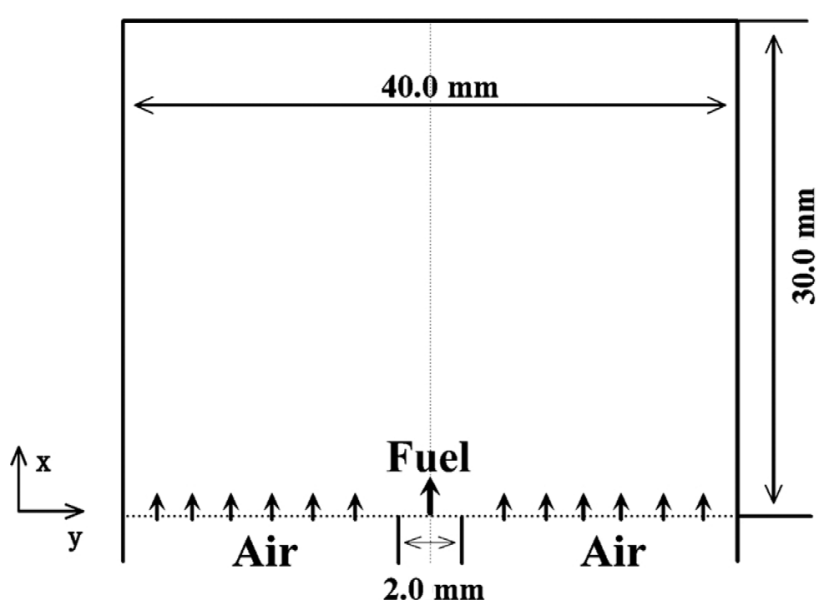

Figure 1: Schematic diagram of the planar jet reactor.

\section{Reduced kinetic mechanism of ethylene}

The reduced mechanism of ethylene (25 species and 131 steps) is obtained from a detailed mechanism of ethylene (71 species and 395 steps) proposed by Wang [9] with the reduction method of error-propagation-based directed relation graph (DRGEP) [7]. Accuracy of the reduced mechanism is examined by comparing laminar flame speed and ignition delay time predicted with the detailed and the reduced mechanisms. Figure 2(a) and (b) give ignition delay time and laminar flame speed obtained

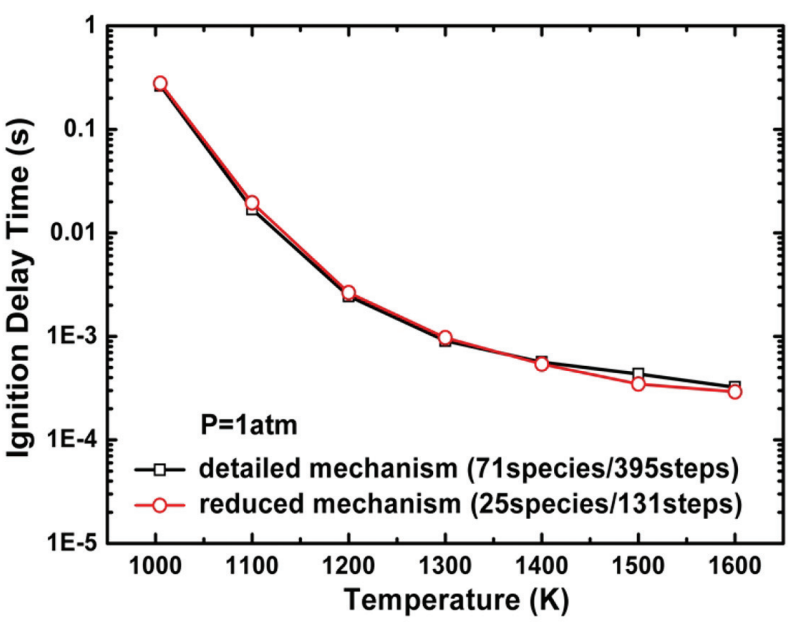

(a)

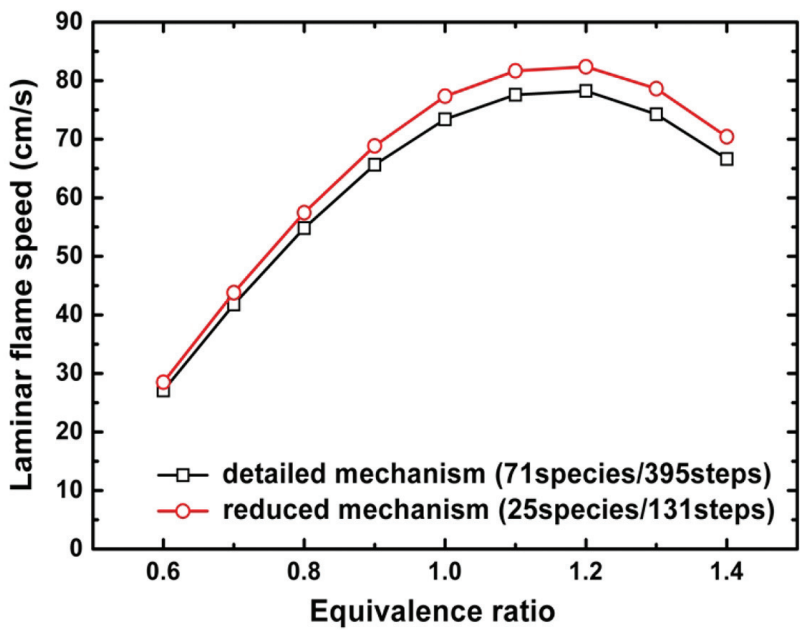

(b)

Figure 2: Comparison of ignition delay time and laminar flame speed obtained by detailed and reduced mechanisms.

with the reduced mechanism and the detailed mechanism. As shown in the figures, good agreements between the two mechanisms can be observed.

\section{Results and discussions}

Figure 3(a) and (b) plot the average temperature profiles at two downstream locations ( $x=6 \mathrm{~mm}, x=18 \mathrm{~mm}$ ). As shown in the figures, satisfactory agreement between the present calculation and the DNS result reported by Yoo et al. [11] can be found. Figure 4 shows contours of instantaneous temperature in the process of fuel ignition and combustion. The dynamic evolution of temperature field due to turbulence mixing and chemical reactions can be easily observed. It is also found from the figure that there exists an ignition delay time of approximately $0.3 \mathrm{~ms}$ for 


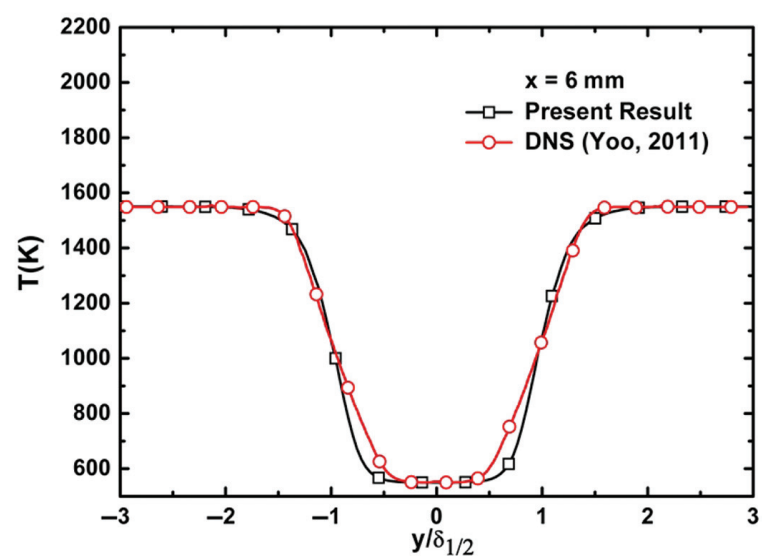

(a)

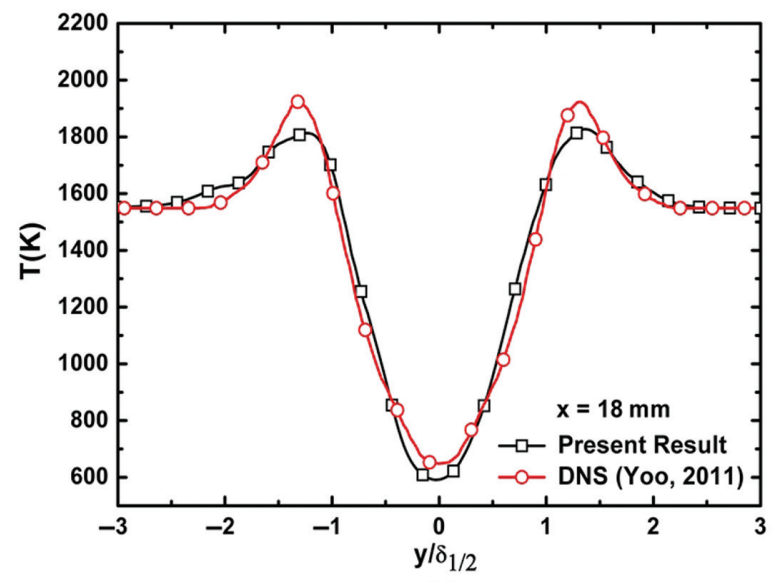

(b)

Figure 3: Comparison of the average temperature profile at varied downstream locations. generation of ethylene flame. Figures 5 and 6 give contours of mass fraction of $\mathrm{OH}$ and $\mathrm{CH}_{2}$ radical at different moments. The formation and consumption of $\mathrm{OH}$ and $\mathrm{CH}_{2}$ are observed to be strongly related to turbulence eddies caused by fuel/air mixing as indicated in Figures 5 and 6 .

\section{Conclusion}

In this paper, unsteady process of ignition and combustion of turbulent plane-jet diffusion flame of ethylene/air is numerically simulated with detached eddy simulation method (DES) and a reduced kinetic mechanism of ethylene consisting of 25 species and 131 steps. The reduced mechanism is gained from a detailed mechanism via DRGEP and agrees well with the detailed mechanism by examining the predicted laminar flame speed and ignition delay time. Ignition and combustion of the ethylene planejet diffusion flame is simulated and dynamic changes of temperature field and $\mathrm{OH}$ mass fraction are obtained. The distributions of Favre-averaged mean temperature profiles at different downstream distances are found to be in good agreement with the DNS results as well as the flame liftedoff height. Dynamic evolutions of temperature field as well as $\mathrm{CH}_{2}$ and $\mathrm{OH}$ radicals show strong relevance with turbulence vortices on the flame formation and propagation. The present numerical study shows that DES method with a qualified reduced mechanism can effectively simulate temporal and spatial evolution of the ignition and combustion process.

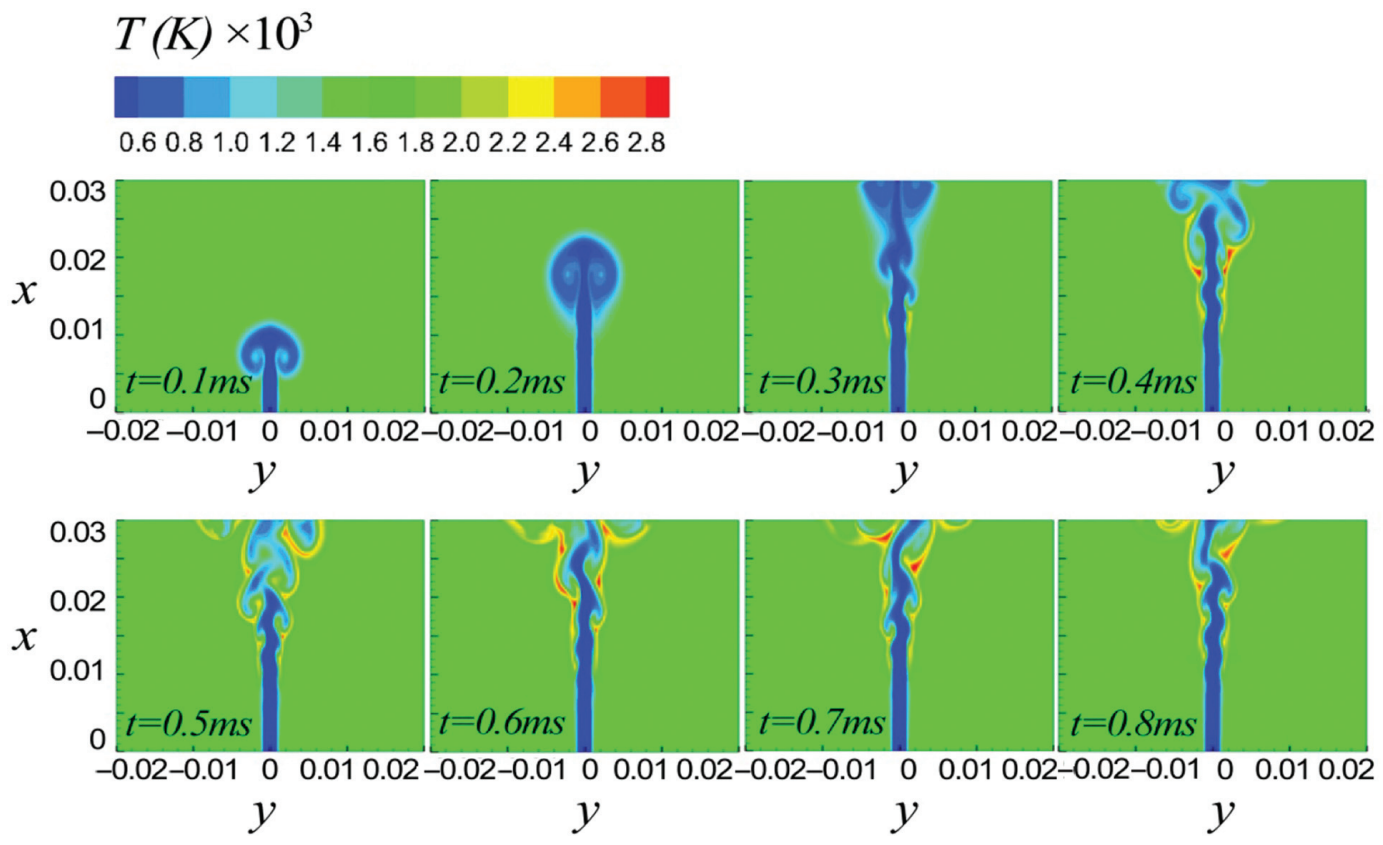

Figure 4: Contours of instantaneous temperature at different times. 
$Y_{O H} \times 10^{-2}$

0.20 .40 .60 .81 .01 .21 .41 .6
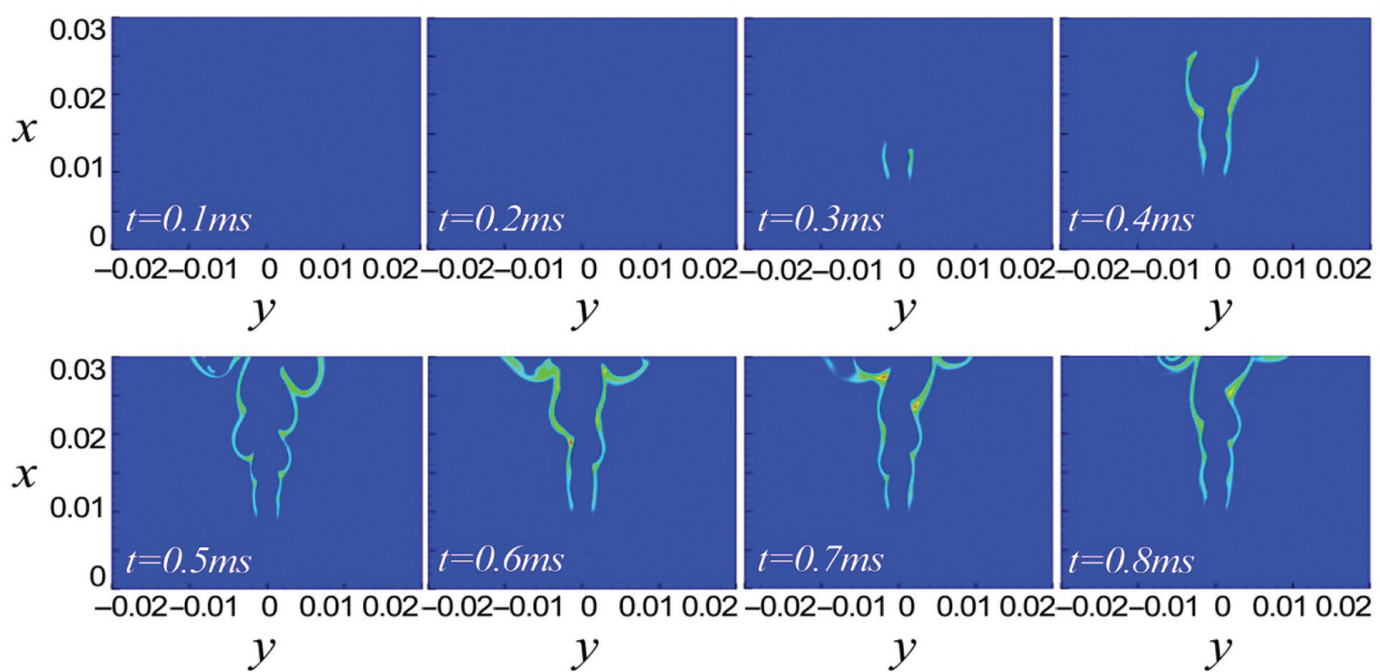

Figure 5: Contours of mass fraction of $\mathrm{OH}$ at different times.

$Y_{\mathrm{CH} 2} \times 10^{-4}$

$\begin{array}{lllllll}0.2 & 0.6 & 1.0 & 1.4 & 1.8 & 2.2 & 2.6\end{array}$

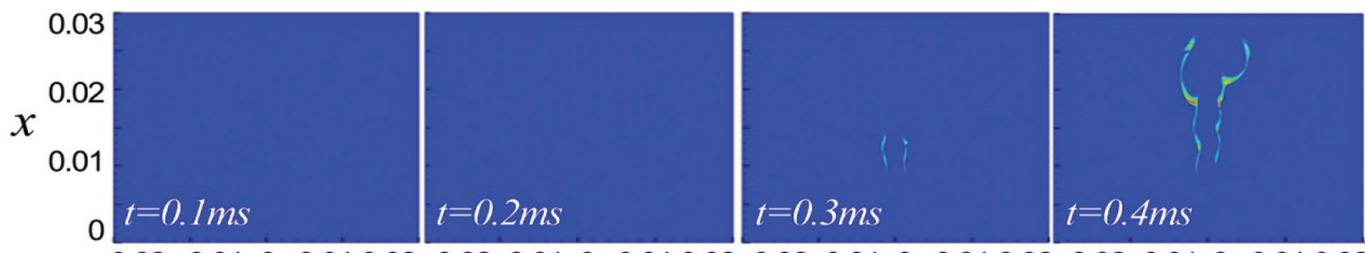

$\begin{array}{llllllllllllllll}-0.02-0.01 & 0 & 0.01 & 0.02-0.02-0.01 & 0 & 0.01 & 0.02-0.02-0.01 & 0 & 0.01 & 0.02-0.02-0.01 & 0 & 0.01 & 0.02\end{array}$ $y=y \quad y \quad y$

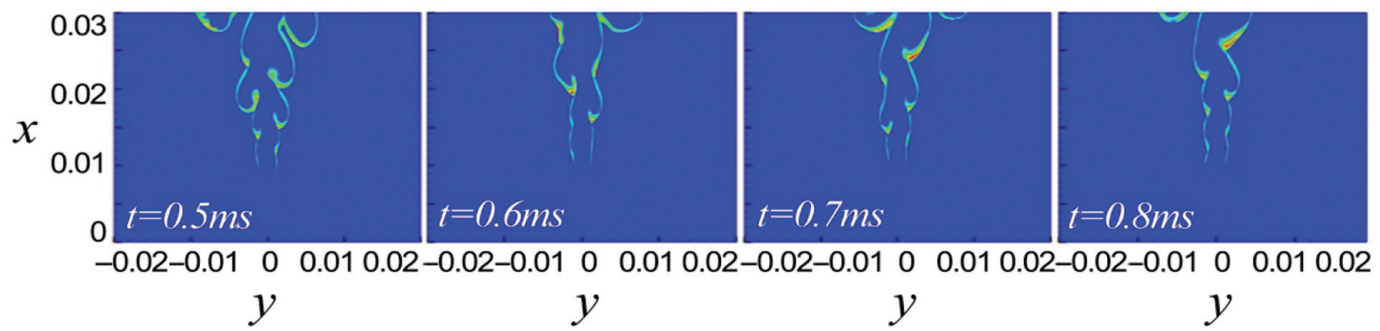

Figure 6: Contours of mass fraction of $\mathrm{CH}_{2}$ at different times. 


\section{Nomenclature}

$T \quad$ average temperature profile

$\delta_{1 / 2} \quad$ local jet half-width

$t \quad$ time

Funding: This study was presented at APCATS 2015 (www.apcats2015.or.kr) and was funded by Natural Science Foundation of China under Contract No. 11172309 and 91441102.

\section{References}

1. Zhong F, Chen L, Chang X. Numerical Study of Ignition and Combustion of Partially Cracked Kerosene in a Mach 2.5 Supersonic Model Combustor. 18th AIAA/3AF International Space Planes and Hypersonic Systems and Technologies Conference, 2012.

2. Montgomery CJ, Zhao W, Tam CJ, Eklund DR, Chen JY. CFD Simulations of a 3-D Scramjet Flameholder Using Reduced Chemical Kinetic Mechanisms. AIAA-2004-3874, 2004:11-14.
3. Das LM. Hydrogen-oxygen reaction mechanism and its implication to hydrogen engine combustion. Int J Hydrogen Energy 1996;21:703-15.

4. Bell JB, Day MS, Grcar JF. Numerical simulation of premixed turbulent methane combustion. Proc Combust Inst 2002;29:1987-93.

5. Lu T, Law CK. A directed relation graph method for mechanism reduction. Proc Combust Inst 2005;30:1333-41.

6. Pepiot-Desjardins $\mathrm{P}$, Pitsch $\mathrm{H}$. An efficient error-propagationbased reduction method for large chemical kinetic mechanisms. Combust Flame 2008;154:67-81.

7. Niemeyer KE, Sung CJ, Raju MP. Skeletal mechanism generation for surrogate fuels using directed relation graph with error propagation and sensitivity analysis. Combust Flame 2010;157:1760-70.

8. Zhong F, Ma S, Zhang X, Sung CJ, Niemeyer KE. Development of efficient and accurate skeletal mechanisms for hydrocarbon fuels and kerosene surrogate. Acta Mech Sin, published on line 21 July, 2015.

9. Wang H, Laskin A. A Comprehensive Kinetic Model of Ethylene and Acetylene Oxidation at High Temperatures. Progress Report for an AFOSR New World Vista Program, 1998.

10. Menter FR. Two-equation eddy-viscosity turbulence models for engineering applications. AIAA J 1994;32:1598-605.

11. Yoo CS, Richardson ES, Sankaran R, Chen JH. A DNS study on the stabilization mechanism of a turbulent lifted ethylene jet flame in highly-heated coflow. Proc Combust Inst 2011;33:1619-27. 